\title{
A 2-piece six-axis force/torque sensor capable of measuring loads applied to tools of complex shapes
}

\author{
Yohan Noh, Lukas Lindenroth, Shuangyi Wang, Richard James Housden, Anne-Sophie van Wingerden, \\ Wanlin Li, Kawal Rhode
}

\begin{abstract}
Measuring forces and torques applied to tools of unconventional shapes can be challenging due to difficulties in connecting commercially available force/torque sensors. We propose an innovative, customizable six-axis force/torque sensor which can be clamped around a tool or end-effector to minimize the proximity between tool tip and sensor structure. The sensor is fabricated using 3d printing technology and consists of two pieces which together form an 8-legged Stewart platform. Each leg is designed as a cantilever beam to allow for a measurable displacement under an external load. The displacements of the legs are measured with 8 light intensity-based optoelectronic sensors, which exhibit high sensitivities and low noise levels without the need for external amplification circuitry. A customized printed circuit board and peripheral hardware are proposed to allow for efficient analog-to-digital conversion of the force/torque measurement. A calibration process is proposed which makes use of a commercial ATI Mini40 sensor and custom hardware to allow for fast calibration routines. Finally, the calibrated sensor design is compared to the ATI Mini40 sensor by measuring sequences of forces and torques, and the maximum errors of force/torque components $\left(F_{x}, F_{y}, F_{z}, M_{x}, M_{y}\right.$, $\left.M_{z}\right)$ are $16.3 \%, \quad 20.0 \%, 27.5 \%, 20.5 \%, 21.6 \%, \quad 14.9 \%$ respectively.
\end{abstract}

\section{INTRODUCTION}

The advent of Robot Technology (RT) has tremendously affected human life with benefits in medical sciences, leisure and service industries as well as industrial automation. Human-robot interaction has become an important challenge as communication between worker or clinician and robotic system through verbal, physical or emotional channels is of paramount importance for the benefits in using a robot. For this purpose, a variety of sensors are employed [1-2].

Industrial robots in manufacturing and factory automation have, for example, assisted in painting an automobile's surface at constant speed, welding components while maintaining a constant force and assemble parts accurately using a combination of force and proximity sensors [3].

Manuscript received March 1st, 2019. The research leading to these results has received funding from the Wellcome Trust IEH Award (Grant No. 102431).

Yohan Noh is with the Department of Biomedical Eng., Division of Imaging Sciences and Biomedical Eng., King's College London, UK., and with the Department of Mechanical and Aerospace Engineering, Brunel University London (e-mail corresponding author: yohan.noh@kcl.ac.uk). Richard James Housden, Shuangyi Wang, and Kawal Rhode are with the
Service and amusement robots with cameras, microphones, force and tactile sensors as well as chemical detectors are designed to work on the street and in service industries where they communicate with people and express their feelings verbally, physically and emotionally (WB or KOBIAN). In particular, physical motions such as gestures and hand shaking improves interactions and communications with people [4-5]. This, however, requires for the physical interaction to be safe. Therefore force or impedance control strategies should be implemented to all robotic joints for the manipulator to simulate joint stiffness, which can in return improve the safety aspect in robot-human interaction settings [6-7].

In medical scenarios, a robot manipulator equipped with a stiffness or highly accurate force/torque sensor can detect tumours, and the force sensors integrated in medical instruments in robot-assisted minimally invasive surgery (MIS) systems can provide force information to the haptic

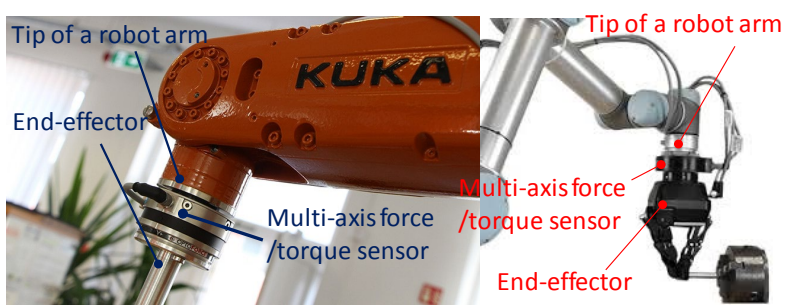

(a)

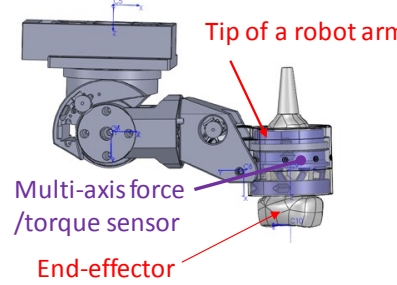

(c) (b)
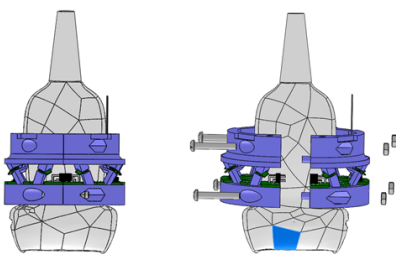

(d)
Figure 1. Commercialised force/torque sensor mounted on an industrial robot manipulator (KUKA) (a) and a collaborative robot manipulator (Universal Robots UR5) (b); (c) Proposed assembly-based six-axis force/torque sensor integrated into a robot arm; (d) Proposed modular assembly-based six-axis force/torque sensor with an ultrasound probe

Department of Biomedical Eng., Division of Imaging Sciences and Biomedical Eng., King's College London, UK

Lukas Lindenroth is with King's College London, School of Natural and Mathematical Sciences, Department of Informatics, UK

Wanlin Li is with Department of Electrical Eng., Queen Mary University of London, UK.

Anne-Sophie van Wingerden with Williams College, Department of Chemistry, Williamstown USA. 
system in the remote side of a master-slave system, thus allowing for a surgeon to identify tissue properties remotely [8-9]. In robot-guided ultrasound applications, measuring and controlling the contact force of the ultrasound probe could presumably help improve image quality and patient comfort.

As explained through the applications above, force/torque sensors are of great importance for human- robot interaction. In this paper, we present the states-of-the-art in force/torque sensing technologies and propose a novel light intensitybased force/torque sensor which can be integrated with any mechanical structures. Conventional sensors are either of cylindrical shape to be mounted directly between components or of ring-like structure to allow user applications such as robot hands or surgical instruments to be attached to the endeffector of the robot arm [10-11]. An additional mechanism has to be designed for the force/torque and the user devices to be integrated to the end-effector, which greatly increases the end-effector size as shown in Figure 1(a) and (b). Besides an impact on the overall size of the end-effector this can lead to the undesired extension of lever arms, which can deteriorate the accuracy and affects the measurement range of the sensor. For this reason, we consider a new force/torque sensor structure which can address above-mentioned issues.

There are various sensing principles through which measuring force/torque components can be addressed. This includes piezoresistive materials [12], strain gauges [13], polyvinylidene fluoride (PVDF) films [14], and Fiber Bragg grating (FBG)[15], conductive rubber, magnet and hall sensors, capacitive sensing, vision-based sensors, light intensity-based fiber-optics or optoelectronic sensors [1617]. The sensing principles mentioned above have been commonly used in many applications. Some sensing elements, in particular piezoresistive materials [12], strain gauges [13], polyvinylidene fluoride (PVDF) films [14], Fiber Bragg grating (FBG)[15] and conductive rubbers, have to be attached to a mechanical flexure to measure deformation directly. The measure of deformation can then be related to the amount of force exerted to the structure. Other sensing principles such as magnetic and hall effect sensing, capacitive sensing, vision as well as light intensity-based fiber optics and optoelectronic sensors do not require physical attachment to the sensor's mechanical flexure. The deformation can be indirectly estimated.

In this paper, we propose a light intensity basedforce/torque sensor using optoelectronics which can be integrated into any mechanical structures and which allows for a high degree of miniaturization. Moreover, the sensor structure can be $3 \mathrm{~d}$ printed which results in a very low fabrication cost [18]. The optoelectronic sensor can measure deformation without mechanical contact to the structure, which makes this sensing approach less prone to mechanical noise. The optoelectronic sensors themselves exhibit low noise levels in comparison with other sensing elements as introduced above. Furthermore, without the need for an amplifier the sensor elements are of high sensitivity.

In this paper, we verify our proposed sensor development approach. A novel six-axis force/torque sensor is designed, fabricated and integrated in an end-effector which is used in a robot-guided medical ultrasound system.

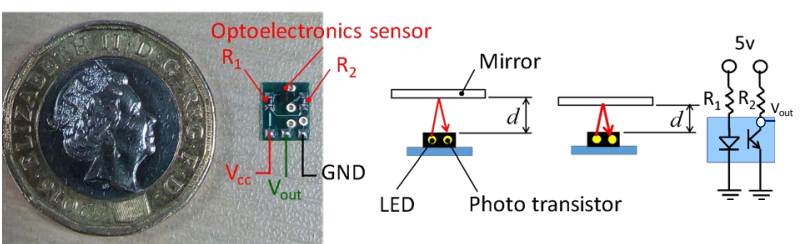

Figure 2. Optoelectronic sensor with two resistors (left) and a pair of an optoelectronic sensor and a mirror to measure a small distance (right).

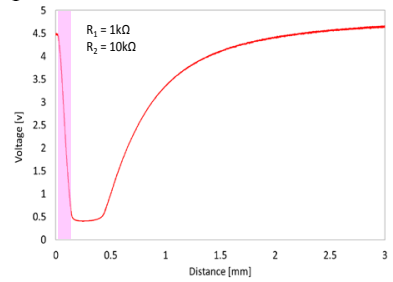

(a)

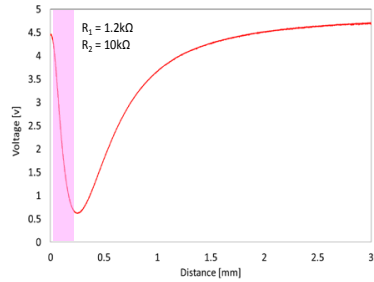

(b)
Figure 3. Experiment results acquired with a motorised linear guide system for obtaining characteristic curves of an optoelectronic sensor according to two different resistor pairs. (a) $\mathrm{R}_{1}=1 \mathrm{k} \Omega$ and $\mathrm{R}_{2}=10 \mathrm{k} \Omega$; (b) $\mathrm{R}_{1}=1.2 \mathrm{k} \Omega$ and $\mathrm{R}_{2}=10 \mathrm{k} \Omega$

\section{FORCE/TORQUE SENSOR}

\section{A. Design Concept}

In this paper, the proposed force/torque sensor is to be integrated in a robot-assisted ultrasound (US) diagnostic system [19]. To attach an US probe to an end-effector with a force/torque sensor, the connection part between the US probe and the force/torque sensor should be designed in such way to minimize its physical footprint. Other requirements to the design include:

1) The proposed sensor can be simply integrated to any mechanical structures (or any end-effector).

2) Optoelectronic sensors are used as a sensing element.

3) Large voltage variation without an amplifier, and lowlevel noise.

4) Simple fabrication, considerably cheaper in comparison with conventional force/torque sensors using different sensing elements as aforementioned above.

To verify the proposed sensor design, a force/torque sensor is fabricated for the use with a medical ultrasound probe (Philips X6-1)[19]. The desired force range, size and sensor material specifications are summarized in Table I.

\section{B. Characteristic curve of optoelectronic sensor}

Optoelectronic sensors detects the presence of reflecting surfaces in their vicinity. Depending on the type of optoelectronic sensor, the distance measurement range varies. In the present force/torque sensing application, the optoelectronic sensor needs to measure the small displacement of a surface in the sensors immediate vicinity. The optoelectronic sensor, NJL5901R-2, $1.0 \times 1.4 \times 0.6 \mathrm{~mm}^{3}$, by New Japan Radio Co., Ltd. (Tokyo, Japan) used in this study consists of two electronic components; an LED and a photo-transistor. When a surface is close to the optoelectronic sensor, the reflected light from the LED is transmitted to the 
TABLE I. Force/Torque Sensor Measurement Ranges, Sensor SIZE AND MATERIAL PROPERTY:

\begin{tabular}{|c|c|c|c|}
\hline $\begin{array}{c}\text { Force } \\
\text { components }\end{array}$ & Force ranges & $\begin{array}{c}\text { Torque } \\
\text { components }\end{array}$ & Torque ranges \\
\hline$F_{x}$ & $+/-10 \mathrm{~N}$ & $M_{x}$ & $+/-500 \mathrm{~N} \cdot \mathrm{mm}$ \\
\hline$F_{y}$ & $+/-10 \mathrm{~N}$ & $M_{y}$ & $+/-500 \mathrm{~N} \cdot \mathrm{mm}$ \\
\hline$F_{z}$ & $+/-10 \mathrm{~N}$ & $M_{z}$ & $+/-1000 \mathrm{~N} \cdot \mathrm{mm}$ \\
\hline \multicolumn{2}{|c|}{ Force/Torque sensor size } & \multicolumn{2}{|c|}{ Material property } \\
\hline \multicolumn{2}{|c|}{} & \multicolumn{2}{|c|}{ Material: polylactide $(\mathrm{PLA})$} \\
Diameter $\emptyset 75.5 \mathrm{~mm}$ & Density: $1.17-1.24\left(\mathrm{~g} / \mathrm{cm}^{3}\right)$ \\
Height: $70.0 \mathrm{~mm}$ \\
(see Figure 4) & $\begin{array}{c}\text { Young's modulus: } 2636(\mathrm{MPa}) \\
\text { Tensile strength: } 46.6(\mathrm{MPa})\end{array}$ \\
\hline
\end{tabular}

photo-transistor, which converts the light intensity variation to a change in the output voltage. Parts of the characteristic curve between the distance and voltage can be approximated with a linear relationship between input and output. The two graphs using two different resistor combinations $R_{1}$ and $R_{2}$ show a linear area presenting the relationship between the output voltage of the optoelectronic sensor and tiny distance $d$ highlighted in purple as shown in Figures 2 and 3.

In general, to guarantee a high-resolution of force/torque sensor, the sensing element should be of high sensitivity. For this reason, we decided to use the purple area in the characteristic curve of the optoelectronic sensor for the force/torque sensor, which is shown in Figure 3(a). This region is of higher linearity and exhibits the desired high sensitivity.

\section{Manufacturing Method and Prototype}

1) Sensor mechanical structure for measuring six-axis force/torque sensor

As aforementioned in the introduction section, the proposed force/torque sensor can be integrated into any mechanical structures or any effectors to reduce the overall space between the force sensor and the tip of the robot arm. For that reason, in contrast to conventional sensor structures which are of cylindrical shape, we propose a sensor structure which can merge two sensing structures together, as shown in Figure 4, to measure six-axis force/torque components. This approach can facilitate any end-effectors to be assembled without taking up a large space on the tip of a robot arm as shown in Figure 1(a) and (b).

The mechanical sensor structure should be designed to be able to house the above-stated optoelectronic sensors in such way to measure the deflection of a part of the sensing structure. Mechanical flexures are commonly arranged to mimic the mechanical structure of a Stewart platform. Stewart platforms are most commonly comprised of six-legs [20-23] and find applications as mechanical sensor structure to measure forces and torques in six degrees of freedom. Such sensors commonly utilize conventional sensing elements such as strain gauges and Fiber Bragg grating (FBG) [13][15]. Those sensing elements require to be attached to the sensor structure. Our approach therefore requires the modification of the conventional Stewart platform.

The modified sensor mechanical flexure is shown in Figure
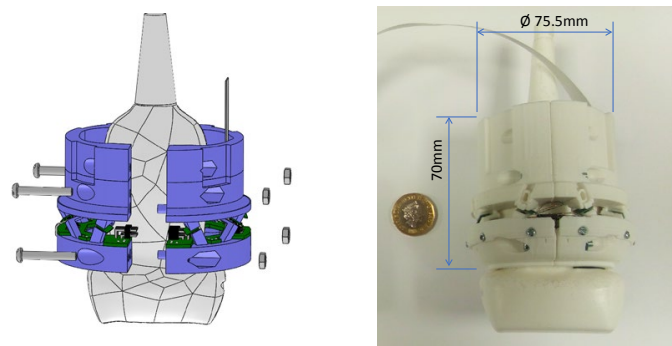

Figure 4. Two-piece sensor structure for measuring six-axis force/torque components (designed in Solidworks).

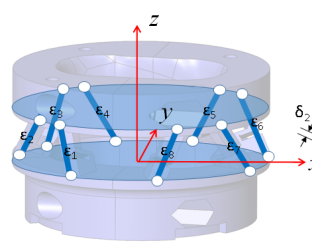

(a)

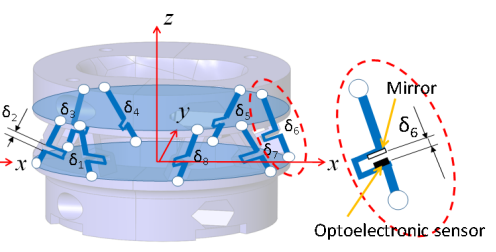

(b)
Figure 5. Modified eight-link Stewart platform: (a) eight strain gauge or FBGs are directly attached on the eight links to measure eight strains $\varepsilon$; (b) eight optoelectronic sensors are mounted on the modified links to measure eight deflections $\delta$.
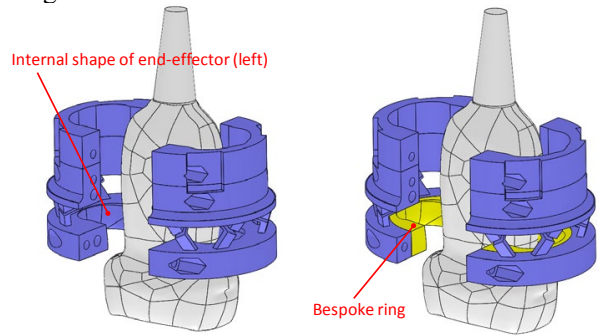

Figure 6. Sensor structure design which can be adapted to fit endeffectors and tools of various sizes and diameters

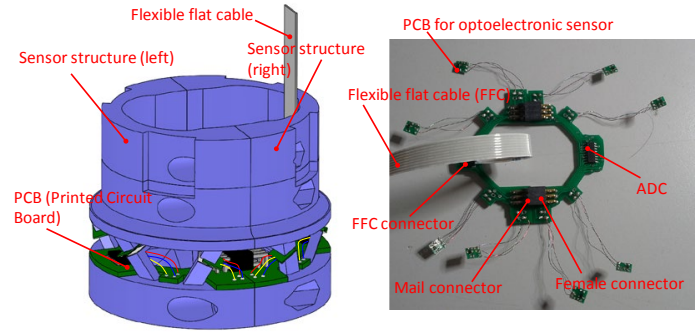

Figure 7. Custom printed circuit board for the proposed six-axis force/torque sensor which includes an ADC, connectors and a flexible flat cable

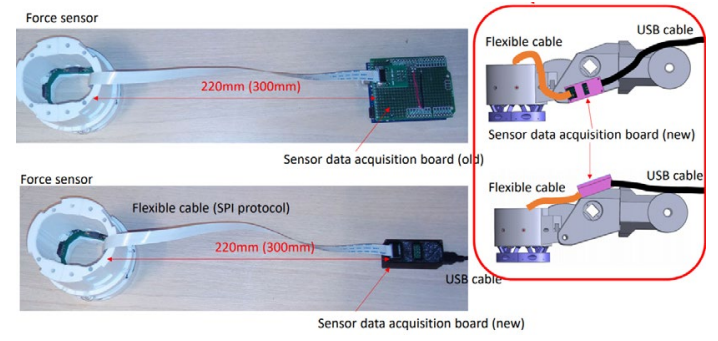

Figure 8. Modular assembly-based six-axis force/torque sensor prototype with data acquisition boards (left) mounted to a robot arm (right)

5(a). It is comprised of eight-legs where each leg has been modified to resemble a cantilever beam. The optoelectronic sensors find place within the leg structures as shown in Figure 5(b). In each leg, a mirror is used as a reflective surface to allow for each optoelectronic sensor to exhibit highly 
sensitive characteristics.

\section{2) Sensor Mechanical structure design for being adapted to any end-effectors}

The two pieces of the sensor structure should be designed to be attachable to end-effectors of differing shapes and size. To ensure adequate an adequate fitting of the end-effector, however, the internal shape of the sensor should be exactly same as the end effector external shape as seen in Figure 6. In this study, an ultrasound probe is used as an end-effector. The ultrasound probe is scanned with an MRI scanner, and its 3D mesh is extracted using ITK-SNAP (http://www.itksnap.org). The mesh model is imported into Solidworks to convert it to an editable CAD model. This approach to developing force/torque sensor structures can be easily adapted to fit different end-effector shapes and offers great flexibility in designing highly integrated force/torque sensors.

\section{3) Printed Circuit Board with $M C U$}

A peripheral circuit for the force/torque sensor is designed to acquire eight optoelectronic sensors, and it comprises of a 12-bit ADC (analog digital converter) MCP3208 (Microchip, USA), four male and female six-pin connectors, and a flexible cable connector. They are mounted on a PCB (printed circuit board) as shown in Figure 7. all the analog data from the optoelectronic sensors are transmitted to the ADC, and the converted digital data are transmitted to an MCU (micro controller) through SPI protocol and the flexible flat cable. Although electrical noise could arise between the optoelectronic sensors and the ADC, at least the electrical noise caused by the cable between the ADC and the MCU can be reduced considerably because the transmitted data between the ADC and the MCU are solely digital.

The proposed flexible flat cable and the cable connector are simply connected to the MCU, and mounted on the robot arm as shown in Figure 8. Whilst universal robot arms are out of control, their abnormal behaviour could damage the force/torque sensor, and vice versa. The flexible flat cable can be replaced when the cable is broken or worn out anytime users want because the force/torque sensor and the transducer are thoroughly separated, and the force/torque sensor can be always protected due to the robots' unpredicted operation because the flexible flat cable is by itself detached when a pulling force is greater than a certain amount of force although I did not test it properly.

\section{MEASURING THEORY}

The measurement frame of the assembled force-torque sensor is set in the origin of the top place, as indicated in Figure 5. The external wrench $\mathbf{w}_{\text {ext }}=\left[\mathbf{f}_{\text {ext }}, \mathbf{m}_{\text {ext }}\right]^{T}$ which is comprised of cartesian forces $\mathbf{f}_{\text {ext }}$ and moments $\mathbf{m}_{\text {ext }}$ is assumed to be acting with respect to this frame.

Each leg of the sensor structure acts as a multidirectional stiffness element of stiffness $\mathbf{K}_{\mathrm{i}}$. The wrench applied to the ith leg of the structure, $\mathbf{w}_{\mathrm{i}}$, can be described by

$$
\mathbf{w}_{\mathrm{i}}=\mathbf{A d}_{\mathrm{i}}^{-1} \cdot \mathbf{w}_{\mathrm{ext}}
$$

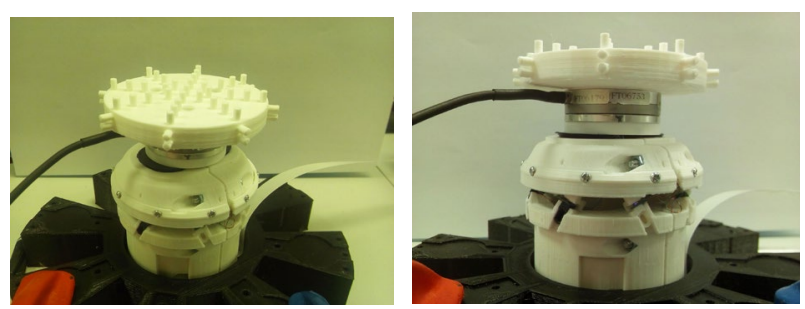

Figure 9. Calibration process with a commercialised force/torque sensor Mini 40 (ATI Industrial Automation, USA)

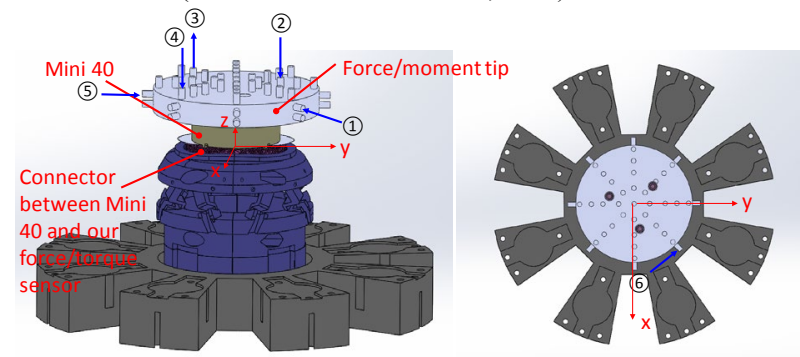

Figure 10. Calibration device for applying different loading conditions to the proposed sensor while measuring the ground truth force using an ATI Mini 40 sensor.

Where Ad is the wrench transformation matrix [18] relating the measurement frame to the tip frame of the i-th leg. The resultant deformation $\Delta \mathbf{x}_{\mathrm{i}}$ of the $\mathrm{i}$-th stiffness element is defined as

$$
\Delta \mathbf{x}_{\mathrm{i}}=\mathbf{K}_{\mathrm{i}}^{-1} \cdot \mathbf{w}_{\mathrm{i}}
$$

Substituting equation (2) in (1) yields

$$
\mathbf{w}_{\mathrm{ext}}=\mathbf{A d}_{\mathrm{i}} \cdot \mathbf{K}_{\mathrm{i}} \cdot \Delta \mathbf{x}_{\mathrm{i}}=\mathbf{G}_{\mathrm{i}} \cdot \Delta \mathbf{x}_{\mathrm{i}}
$$

As the legs of the sensor structure are designed in such way to only allow for axial deflection, matrix $\mathbf{G}_{\mathbf{i}}$ will, in theory, only contain one column with non-zero elements $\widehat{\mathbf{G}}_{\mathrm{i}}$. Due to the parallel nature of the structure, the non-zero columns can therefore be arranged in the form

$$
\begin{aligned}
& \mathbf{w}_{\text {ext }}=\left[\widehat{\mathbf{G}}_{1}, \widehat{\mathbf{G}}_{2}, \widehat{\mathbf{G}}_{3}, \widehat{\mathbf{G}}_{4}, \widehat{\mathbf{G}}_{5}, \widehat{\mathbf{G}}_{6}, \widehat{\mathbf{G}}_{7}, \widehat{\mathbf{G}}_{8}\right] \\
& \cdot\left[\delta_{1}, \delta_{2}, \delta_{3}, \delta_{4}, \delta_{5}, \delta_{6}, \delta_{7}, \delta_{8}\right]^{T}=\mathbf{M} \cdot \boldsymbol{\delta}
\end{aligned}
$$

With $\delta_{\mathrm{i}}, \mathrm{i} \in[1,8]$ being the axial displacement of the $\mathrm{i}$-th leg. The matrix $\mathbf{M}$ can be obtained through the calibration of the sensor.

\section{Sensor Calibration And Sensor Performance}

The sensor is calibrated to determine the relationship between its input forces and moments and the corresponding output voltages of the individual optoelectronic sensors. In a conventional calibration process different physical loads are applied to the sensor structure while the sensor's output voltages are measured. The recorded values are then used to calculate the matrix M. To fully define the decoupled matrix $\mathbf{M}$, this, however, requires a large number of input-output sets as well as a very precise and accurate knowledge of the applied load. Calibrating the sensor structure with a designated calibration device and a set of weights can be time- 
consuming and difficult due to friction occurring on pulleys and between weight pulling wires and the wire guides [24].

Another possible calibration process is to use a robot arm, a simple calibration device and a set of weights which are mounted on the calibration device. The robot arm then rotates the sensor along its three principal axis to achieve different force/torque conditions [25]. To guarantee a high precision calibration, however, it is difficult to compensate for dynamic forces and moments which are applied to the sensor structure, as the ground truth force and torque magnitudes cannot be measured.

In this work we are proposing a calibration methodology which can be carried out easily. For the calibration process, a commercialized force/torque sensor (ATI Mini40, ATI Industrial Automation, USA) with a force/torque tip is mounted on our six-axis force/torque sensor as shown in Figure 9. The force/moment tip is designed in such way as to be able to apply a variety of different force/torque conditions as shown in Table II. The output voltages of the six optoelectronic sensors are acquired via the above mentioned sensor peripherals. An Arduino UNO is used as an MCU. The ground-truth forces and moments are measured by the ATIMini 40 with a $100 \mathrm{~Hz}$ sampling rate. An example of different load measurements is shown in Figures 9-10. From the recorded data, the calibration matrix can be calculated using Least Squares Regression method [26]. The calculated calibration matrix is shown in Eq. 5.
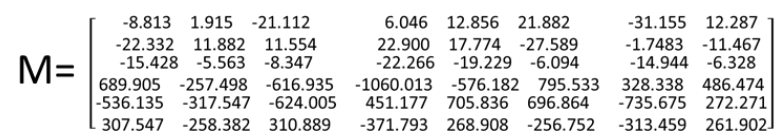

To verify the calibration matrix, another force/torque tip is mounted on the ATI Mini 40 and the proposed force/torque sensor in series as shown in Figure 9. External force/torque components are applied and the ground-truth values from the ATI Mini 40 are compared to the estimated ones. The latter are obtained using the aforementioned calibration matrix at a sampling rate of $100 \mathrm{~Hz}$. A comparison of the estimated
TABLE II. APPLIED ForCE/TORQUE CONDITIONS ON THE FORCE/MOMENT TIP:

\begin{tabular}{|c|c|c|c|}
\hline $\begin{array}{c}\text { Applied } \\
\text { force/torque }\end{array}$ & $\begin{array}{c}\text { Force/Torque } \\
\text { condition }\end{array}$ & $\begin{array}{c}\text { Applied } \\
\text { force/torque }\end{array}$ & $\begin{array}{c}\text { Force/Torque } \\
\text { condition }\end{array}$ \\
\hline (1) & $\begin{array}{l}-\mathrm{F}_{\mathrm{x}},-\mathrm{F}_{\mathrm{y}} \\
+\mathrm{T}_{\mathrm{x}},-\mathrm{T}_{\mathrm{y}}\end{array}$ & (4) & $\begin{array}{c}-\mathrm{F}_{\mathrm{z}}, \\
+\mathrm{T}_{\mathrm{x}},+\mathrm{T}_{\mathrm{y}},\end{array}$ \\
\hline (2) & $\begin{array}{l}-F_{z}, \\
-T_{x}\end{array}$ & (5) & $\begin{array}{l}+\mathrm{F}_{\mathrm{y}} \\
-\mathrm{T}_{\mathrm{x}}\end{array}$ \\
\hline (3) & $\begin{array}{c}+\mathrm{F}_{\mathrm{z}} \\
-\mathrm{T}_{\mathrm{x}},+\mathrm{T}_{\mathrm{y}},\end{array}$ & (6) & $\begin{array}{c}+\mathrm{F}_{\mathrm{x}},+\mathrm{F}_{\mathrm{y}} \\
-\mathrm{T}_{\mathrm{x}},-\mathrm{T}_{\mathrm{y}},+\mathrm{T}_{\mathrm{z}},\end{array}$ \\
\hline
\end{tabular}

TABLE III. PROPOSED ForCE/TORQUE SENSOR ACCURACY:

\begin{tabular}{|c|c|c|c|}
\hline $\begin{array}{c}\text { Force/Torque } \\
\text { components }\end{array}$ & $\begin{array}{c}\text { Force/Torque } \\
\text { measurable } \\
\text { ranges }\end{array}$ & $\begin{array}{c}\text { Maximum } \\
\text { Error }\end{array}$ & RSM Error \\
\hline$F_{x}$ & $+/-10.0 \mathrm{~N}$ & $16.35 \%$ & $0.45 \mathrm{~N}$ \\
\hline$F_{y}$ & $+/-10.0 \mathrm{~N}$ & $20.08 \%$ & $0.44 \mathrm{~N}$ \\
\hline$F_{z}$ & $+/-10.0 \mathrm{~N}$ & $27.51 \%$ & $0.51 \mathrm{~N}$ \\
\hline$M_{x}$ & $+/-500.0 \mathrm{~N} \cdot \mathrm{mm}$ & $20.54 \%$ & $17.67 \mathrm{~N} \cdot \mathrm{mm}$ \\
\hline$M_{y}$ & $+/-500.0 \mathrm{~N} \cdot \mathrm{mm}$ & $21.62 \%$ & $18.40 \mathrm{~N} \cdot \mathrm{mm}$ \\
\hline$M_{z}$ & $+/-1000.0 \mathrm{~N} \cdot \mathrm{mm}$ & $14.94 \%$ & $13.10 \mathrm{~N} \cdot \mathrm{mm}$ \\
\hline
\end{tabular}

forces and torques with the ground truths is shown in Figure 10. The proposed force/torque sensor can estimate the ground-truth values considerably well despite some large deviations as shown Figure 10 and Table III. The RMS errors $\left(F_{x}, F_{y}, F_{z}, M_{x}, M_{y}, M_{z}\right)$ show $0.45 \mathrm{~N}, 0.44 \mathrm{~N}, 0.51 \mathrm{~N}$, $17.67 \mathrm{~N} \cdot \mathrm{mm}, 18.40 \mathrm{~N} \cdot \mathrm{mm}$, and $13.10 \mathrm{~N} \cdot \mathrm{mm}$ respectively. Although the new calibration approach cannot show linearity, repeatability, and hysteresis, overall calibration time is very short, and the calibration process is very simple. From this experiment results, the proposed calibration process and the proposed force/torque sensor are well-verified.

\section{DISCUSSION}

There are various reasons which contribute to the larger deviations between the estimated forces and torques and the ground truth magnitudes. The sensor structure is fabricated from polylactide (PLA) filament. The material itself exhibits largely hysteretic behavior. As hysteresis is not considered in the calibration, the caused deviations are propagated to the

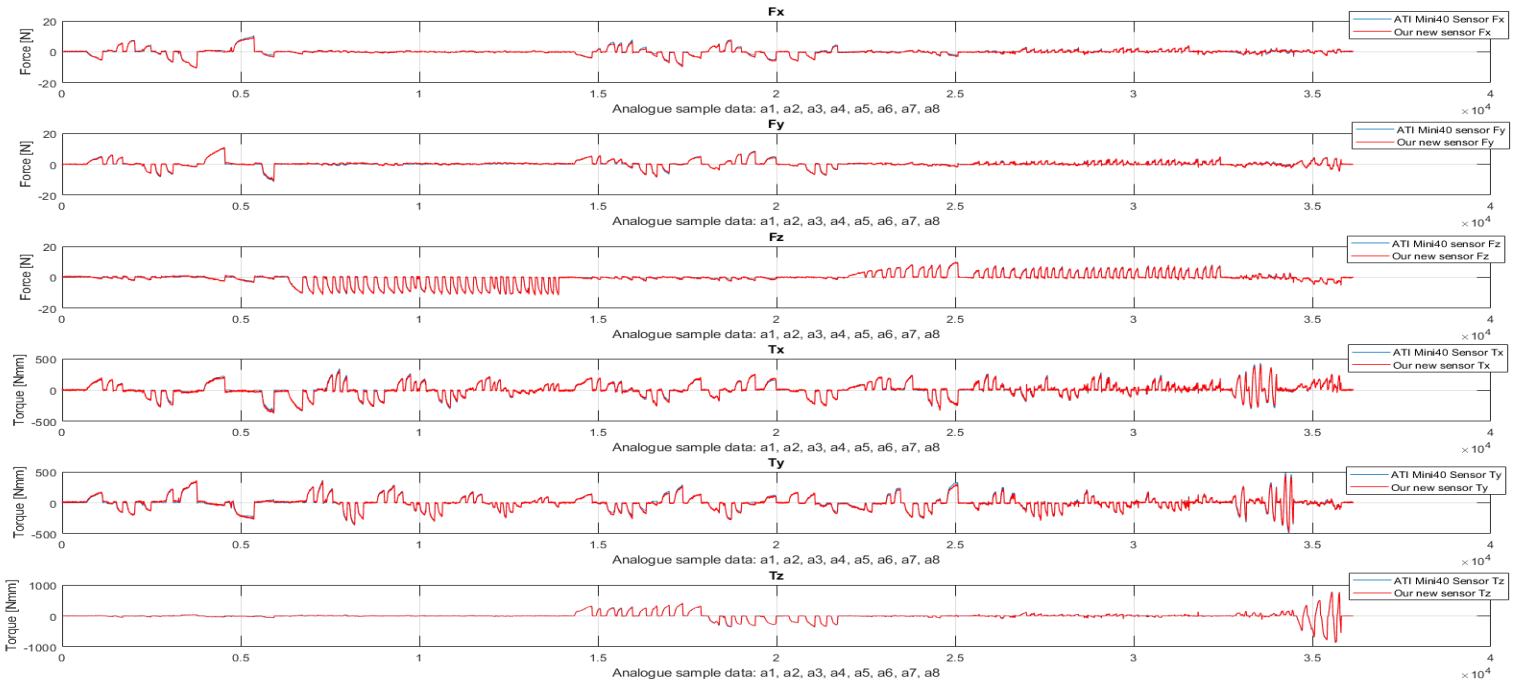

Figure 11. accuracy comparison between Mini 40 and our force/torque sensor 
subsequent force/torque estimations. To reduce hysteresis in the sensor structure, different $3 \mathrm{~d}$ printing materials can be chosen. A selective laser-sintered metal structure, for instance, could help greatly reduce hysteresis effects while still be manufacturable at a fraction of the cost of a sensor structure fabricated through $\mathrm{CNC}$ machining, wire electric discharge machining (WEDM), or electric discharge machining (EDM).

Although we optimised the two resistors which define the sensitivity of the optoelectronic sensors, this could be improved to suit the required measurement range better. Furthermore, the achieved deflection of $0.1 \mathrm{~mm}$ is still comparably large. The use of a metal structure could help reduce this limitation.

\section{CONCLUSION AND FUTURE WORKS}

In this paper, we proposed a new six-axis force/torque sensor which can be disassembled to fit various shapes and sizes of mechanical end-effectors. We contributed the following: 1) We have derived a novel 2-piece sensor structure 2) We have shown the applicability of optoelectronic sensors for the given sensor structure to obtain a highly sensitive and low-noise force/torque measurement. 3) We proposed an efficient way to calibrate the sensor using a purposefully-designed calibration device. 4) We verified the sensor design and its calibration with a commercial force/torque sensor.

As part of future investigations, a metal sensor structure will be fabricated to reduce the hysteresis problem and the sensitivity of the optoelectronic sensor will be further optimized. Furthermore, the footprint of the sensor structure will be miniaturised and its force/torque measurement range adapted by modifying the dimensions of mechanical flexures as well as by the choice of 3d-printed material.

\section{REFERENCES}

[1] Yu Qing-xiao, Yuan Can, Fu Zhuang and Zhao Yan-zheng, "Research of the Localization of Restaurant Service Robot", International Journal of Advanced Robotic Systems, Vol. 7, No. 3, pp. 227-238, 2010.

[2] Hiroyasu Miwa, Kazuko Itoh, Munemichi Matsumoto, Massimiliano Zecca, Hideaki Takanobu, Stefano Roccella, Maria Chiara Carrozza, Paolo Dario, Atsuo Takanishi,"Effective Emotional Expressions with Emotion Expression Humanoid Robot WE-4RII - Integration of Humanoid Robot Hand RCH-1 _", 2011 IEEE/RSJ International Conference on Intelligent Robots and Systems (IROS), pp. 2203 - 2208, 2011.

[3] Ijeoma W. Muzan, Tarig Faisal, H. M. A. A. Al-Assadi, Mahmud Iwan, "Implementation of Industrial Robot for Painting Applications, " Procedia Engineering, vol.41, pp. 1329-1335, 2012.

[4] Tatsuhiro Kishi, Souichiro Shimomura, Hajime Futaki, Hiroshi Yanagino, Sarah Cosentino, Takashi Nozawa, Kenji Hashimoto and Atsuo Takanishi, "Development of a Humorous Humanoid Robot Capable of Quick-and-Wide Arm Motion," IEEE Robotics and Automation Letters, Vol. 1, Iss. 2, pp. 1081-1088, 2016.

[5] João Avelino, Filipa Correia, João Catarino, Pedro Ribeiro, Plinio Moreno, Alexandre Bernardino, and Ana Paiva," The Power of a Handshake in Human-Robot Interactions," 2018 IEEE/RSJ International Conference on Intelligent Robots and Systems (IROS), pp. 1864-1869, 2018.

[6] T. Asfour, L. Kaul, M. Wächter, S. Ottenhaus, P. Weiner, S. Rader, R. Grimm, Y. Zhou, M. Grotz, F. Paus, D. Shingarey, and H. Haubert, “ ARMAR-6: A Collaborative Humanoid Robot for Industrial Environments,“ IEEE/RAS International Conference on Humanoid Robots (Humanoids), pp. 447-454, 2018.
[7] Sheng-Yen Lo, Ching-An, Cheng, Han-Pang Huang," Virtual Impedance Control for Safe Human-Robot Interaction," Journal of Intelligent \& Robotic Systems, vol.82, Iss.1, pp 3-19, 2016.

[8] Min Li, Hongbin Liu, Allen Jiang, Lakmal D Seneviratne, Prokar Dasgupta, Kaspar Althoefer, Helge Wurdemann, "Intra-operative tumour localisation in robot-assisted minimally invasive surgery: A review, “ Proc Inst Mech Eng H., 228(5), pp. 509-522, 2014.

[9] Rozaliia F. Solodova, Vladimir V. Galatenko, Eldar R. Nakashidze, Sergey G. Shapovalyants, Igor L. Andreytsev, Mikhail E. Sokolov, and Vladimir E. Podolskii, "Instrumental Mechanoreceptoric Palpation in Gastrointestinal Surgery," Minim Invasive Surg., 2017

[10] ATI Industrial Automation, Multi-axis Force/Torque Sensors. Available online: http://www.ati-ia.com/products/ft/sensors.aspx (accessed on 1 March 2019).

[11] OnRobot, Industrial Automation, Multi-axis Force/Torque Sensors. Available online: https://onrobot.com/en/products/hex-force-torquesensor (accessed on 1 March 2019).

[12] D.V. Dao, T. Toriyama, J. Wells, S. Sugiyama, "Silicon piezoresistive six-degree of freedom micro force-moment sensor," Sens. Mater., 15, pp. 113-135, 2003.

[13] U. Seibold, B. Kubler, G. Hirzinger, "Prototype of instrument for minimally invasive surgery with 6-axis force sensing capability," In Proceedings of the IEEE International Conference on Robotics and Automation (ICRA) 2005.

[14] Y. Jia, X. Chen, Q. Ni, L. Li, C. Ju, "Dependence of the impact response of polyvinylidene fluoride sensors on their supporting materials' elasticity, "Sensors, 13, pp.8669-8678, 2013.

[15] C. J. Pacheco, A.C. Bruno, "A noncontact force sensor based on a fibre bragg grating and its application for corrosion measurement, "Sensors, 13, pp. 11476-11489, 2013.

[16] Y. Noh, S. Sareh, H. Würdemann, H. Liu, J. Housden, K. Rhode, K. Althoefer, "Three-axis fiber-optic body force sensor for flexible manipulators," IEEE Sensors Journal, vol.16, pp. 1641-1651, 2016.

[17] Yohan Noh, Joao Bimbo, Sina Sareh, Helge Wurdemann, Jan Fras, Damith Suresh Chathuranga, Hongbin Liu, James Housden, Kaspar Althoefer and Kawal Rhode, "Multi-Axis Force/Torque Sensor Based on Simply-Supported Beam and Optoelectronics, "Sensors, 16, 1936, 2016.

[18] Jiantao Yao, Hongyu Zhang, Ximei Xiang, Huidong Bai, Yongsheng Zhao, "A 3-D printed redundant six-component force sensor with eight parallel limbs, "Sensors and Actuators A: Physical, A 247, pp. 90-97, 2016.

[19] Shuangyi Wang, James Housden, Yohan Noh, Anisha Singh, Junghwan Back, Lukas Lindenroth, Hongbin Liu, Joseph Hajnal, Kaspar Althoefer, Davinder Singh, Kawal Rhode, "Design and Implementation of a Bespoke Robotic Manipulator for Extra-corporeal Ultrasound," Journal of Visualized Experiments, 2019.

[20] T.A. Dwarakanath, T.K. Bhaumick, D. Venkatesh, " Implementation of Stewart platform based force-torque sensor," In Proceedings of the IEEE International Conference on Multisensor Fusion and Integration for Intelligent Systems (MFI), pp.32-36, 1999.

[21] R Ranganath, P. S. Nair, T. S. Mruthyunjaya, A. Ghosald, "A forcetorque sensor based on a Stewart Platform in a near-singular configuration, "Mechanism and Machine Theory, vol.39, Iss.9, pp. 971998, 2004.

[22] J. S. Dai, D. R. Kerr, "A six-component contact force measurement device based on the Stewart platform, "ARCHIVE Proceedings of the Institution of Mechanical Engineers Part C Journal of Mechanical Engineering Science 1989-1996 (vols 203-210) 214(5):687-697.

[23] R. Haslinger, P. Leyendecker, U. Seibold, "A fiberoptic force-torquesensor for minimally invasive robotic surgery, "In Proceedings of the IEEE International Conference on Robotics and Automation (ICRA) 2013.

[24] Min-Kyung Kang, Soo bum Lee, Jung-Hoon Kim, "Shape ptimization of a mechanically decoupled six-axis force/torque sensor," Sensors and Actuators A: Physical vol.209, pp. 41-51, 2014.

[25] Lars Richter, Ralf Bruder, Achim Schweikard, "Calibration of Force/Torque and Acceleration for an Independent Safety Layer in Medical Robotic Systems, “ cureus.59, 2012.

[26] L.L. Nathans, F.L. Oswald, K. Nimon, "Interpreting multiple linear regression: A guidebook of variable importance," Pract. Assess. Res. Eval. vol.17, pp. 1-19, 2012. 